Konrad-Zuse-Zentrum für Informationstechnik Berlin

RALF BORNDÖRFER ROBERT WEISMANTEL

\title{
Set Packing Relaxations of Some Integer Programs
}




\title{
Set Packing Relaxations of Some Integer Programs
}

\author{
Ralf Borndörfer $\quad$ Robert Weismantel*
}

\begin{abstract}
This paper is about set packing relaxations of combinatorial optimization problems associated with acyclic digraphs and linear orderings, cuts and multicuts, and set packings themselves. Families of inequalities that are valid for such a relaxation as well as the associated separation routines carry over to the problems under investigation.
\end{abstract}

Mathematics Subject Classification (MSC 1991). 90C10

\section{Introduction}

This paper is about relaxations of certain combinatorial optimization problems in the form of a set packing problem and the use of such relaxations in a polyhedral approach.

The set packing or stable set problem (SSP) is to find, in a graph $G=(V, E)$ with node weights $c$, a set packing or stable set, i.e., a set of pairwise non-adjacent nodes, of maximum weight. Set packing problems are among the best studied combinatorial optimization problems with beautiful theories connecting this area of research to Fulkerson's antiblocking theory, the theory of perfect graphs, perfect and balanced matrices, semidefinite programming, and other fields, see Grötschel, Lovász \& Schrijver [1988] for a survey. Likewise, the set packing polytope, i.e., the convex hull of all set packings of a graph, plays a prominent role in polyhedral combinatorics not only because large classes of (facet defining) inequalities are known. Perhaps even more important, many of them can be separated in polynomial time. In particular, odd cycle, orthonormal representation, and (superclasses of) odd antihole constraints are polynomial time separable, see again Grötschel, Lovász \& Schrijver [1988] and Lovász \& Schrijver [1991].

Our aim in this paper is to transfer some of these results to other combinatorial optimization problems. We show that the acyclic subdigraph and the linear ordering problem, the max cut, the $k$-multicut, and the clique partitioning problem, and the set packing problem itself have interesting combinatorial relaxations in form of a set packing problem. Families of inequalities that are valid for these relaxations and the associated separation routines carry over to the problems under investigation. The procedure is an application of a more general method to construct relaxations of combinatorial optimization problems by means of affine transformations that we discuss in a forthcoming paper. This method is in the tradition of projection techniques such as Balas \& Pulleyblank [1989], Pulleyblank \& Shepherd [1993], and Chopra \& Rao [1994a,b] and, in particular, the approach of Padberg \& Sung [1991] to use affine transformations for the comparison of TSP formulations.

The paper is subdivided into four parts. Section 2 lists notation and results on set packing for future reference. It also recalls three earlier frameworks that give results similar, yet not identical, to ours; we shall point out similarities and differences throughout the article. Section 3 is devoted to a study of the acyclic subdigraph and the linear ordering problem, see Grötschel, Jünger \& Reinelt [1985b,a]. A main result in this section is that a class of Möbius ladders with dicycles of arbitrary length belong to a (larger) class of odd cycles of an appropriate set packing relaxation; this superclass is polynomial time separable. Section 4 deals with set packing relaxations of the clique partitioning, the $k$-multicut, and the max cut problem, see Grötschel \& Wakabayashi [1990] and Deza \& Laurent [1997]. We introduce a class of "inequalities from odd cycles of lower triangle inequalities" that contains the 2-chorded cycle inequalities. Section 5 treats the set packing problem itself. We show, in particular, that the wheel inequalities of Barahona \& Mahjoub [1994] and Cheng \& Cunningham [1997] are odd cycle inequalities of a suitable set packing relaxation. We also introduce a new family of facet defining inequalities for the set packing polytope: The "cycle of cycles" inequalities. This class can be separated in polynomial time.

${ }^{*}$ Supported by the Gerhard Hess Forschungsförderpreis of the Deutsche Forschungsgemeinschaft, the Kultusministerium Sachsen Anhalt, and an EU Donet Project. 


\section{Terminology}

The subsequent sections resort to the following notation and results about the set packing problem. The set packing problem for a graph $G$ with node weights $c$ can be formulated as an integer program

$$
\max c^{T} x \quad A x \leq \mathbf{1}, \quad x \in\{0,1\}^{V},
$$

where $A=A(G) \in\{0,1\}^{E \times V}$ is the edge-node incidence matrix of $G$ and $\mathbf{1}$ a vector of all ones of compatible dimension. Associated to this program is the stable set polytope

$$
P_{\mathrm{SSP}}:=\operatorname{conv}\left\{\chi^{S}: S \text { is a stable set in } G\right\}=\operatorname{conv}\left\{x \in\{0,1\}^{n}: A x \leq \mathbf{1}\right\},
$$

the convex hull of all incidence vectors of stable sets in $G$ or, equivalently, of all solutions of (SSP). Occasionally, we will denote this polytope also by $P_{\mathrm{SSP}}(G)$. For technical reasons, we will actually not work with the stable set polytope $P_{\mathrm{SSP}}$ itself, but with its antidominant

$$
\breve{P}_{\mathrm{SSP}}:=P_{\mathrm{SSP}}-\mathbb{R}_{+}^{V}=\left\{x \in \mathbb{R}^{V}: \exists y \in P_{\mathrm{SSP}}: x \leq y\right\} .
$$

This construction allows to consider vectors with arbitrary negative coordinates without destroying the polyhedral structure of $P_{\mathrm{SSP}}$ : Obviously, the valid inequalities for $\breve{P}_{\mathrm{SSP}}$ are exactly the valid inequalities for $P_{\mathrm{SSP}}$ of the form $a^{\mathrm{T}} x \leq \alpha$ with non-negative coefficients. Since the stable set polytope $P_{\mathrm{SSP}}$ is downmonotone, its non-trivial valid inequalities all have non-negative coefficients. We can thus work with $\breve{P}_{\text {SSP }}$ as well as with $P_{\mathrm{SSP}}$.

We list some terminology to state five fundamental results on $P_{\mathrm{SSP}}$ (or $\check{P}_{\mathrm{SSP}}$ ). A clique in a graph $G$ is a set of pairwise adjacent nodes. A walk is a sequence $v_{1}, e_{1}, v_{2}, e_{2}, \ldots, e_{k}, v_{k+1}$ of nodes $v_{i}$ and edges $e_{i}$ such that $e_{i}=v_{i} v_{i+1}, i=1, \ldots, k$. A closed walk has $v_{1}=v_{k+1}$. A walk is a path if all its nodes are different, except possibly the first and the last, which can be identical; in this case, the path is called a cycle. The analogous concepts for digraphs, with the additional stipulation that all arcs have to be "oriented in the same direction", are called diwalk, closed diwalk, dipath, and dicycle, respectively. A (di)walk (and hence a (di)path or (di)cycle) is odd if $k$ is odd, i.e., if it contains an odd number of nodes, even otherwise. An edge that joins two nodes of a cycle, but is not a member of the cycle, is a chord. A 2-chord is a chord of the form $v_{i} v_{i+2}$ (indices $>k$ taken modulo $k$ ). A chordless cycle is a hole. For convenience of notation, we will occasionally consider (di)paths and (di)cycles as sets of nodes, edges, or arcs, and we will denote edges as well as arcs with the symbols $i j$ and $(i, j)$; the latter will be used in cases like $(i, i+1)$. Finally, $\operatorname{supp}(x)=\left\{i \in V: x_{i} \neq 0\right\}$ is the support of a vector $x \in \mathbb{R}^{V}$.

The results on $P_{\mathrm{SSP}}$ that we need are summarized in the following two theorems; we state them for $\breve{P}_{\mathrm{SSP}}$.

\subsection{Theorem (Padberg [1973], Grötschel, Lovász \& Schrijver [1988])}

Let $G=(V, E)$ be a graph and $\breve{P}_{\mathrm{SSP}}$ the antidominant of the associated set packing polytope.

(i) If $Q$ is a clique in $G$, the clique inequality $\sum_{i \in Q} x_{i} \leq 1$ is valid for $\breve{P}_{\mathrm{SSP}}$; it is facet defining if and only if $Q$ is a maximal clique (with respect to set inclusion).

(ii) If $C$ is an odd cycle in $G$, the odd cycle inequality $\sum_{i \in C} x_{i} \leq(|C|-1) / 2$ is valid for $\breve{P}_{\text {SSP. }}$.

(iii) Let $u \in \mathbb{R}^{V}$ be an orthonormal representation of $G$, i.e., $\left|u_{i}\right|=1$ for all $i \in V$ and $u_{i}^{\mathrm{T}} u_{j}=0$ holds for all ij $\notin E$, and let $c \in \mathbb{R}^{V}$ be an additional arbitrary vector with $|c|=1$. The orthonormal representation inequality $\sum_{i \in V}\left(c^{\mathrm{T}} u_{i}\right)^{2} x_{i} \leq 1$ is valid for $\breve{P}_{\text {SSP. }}$.

Separation of clique inequalities is $\mathcal{N} \mathcal{P}$-hard. But the clique inequalities belong to the more general class of orthonormal representation inequalities which can be separated in polynomial time.

\subsection{Theorem (Grötschel, Lovász \& Schrijver [1988])}

Let $G=(V, E)$ be a graph, $\breve{P}_{\mathrm{SSP}}$ the antidominant of the associated set packing polytope, and $x \in \mathbb{Q}^{V}$. Suppose that $x_{i}+x_{j} \leq 1$ holds for all edges $i j \in E$. Then:

(i) Orthonormal representation inequalities violated by $x$ can be separated in polynomial time.

(ii) Odd cycle inequalities violated by $x$ can be separated in polynomial time. 
The literature has three frameworks that give results similar to this article:

- The independence system approach, see Nemhauser \& Trotter [1973], Sekiguchi [1983], Euler, Jünger \& Reinelt [1987], Laurent [1989], Nobili \& Sassano [1989], and others.

- The transitive packing approach of Müller [1996], Müller \& Schulz [1995, 1996], Schulz [1996].

- The $\left\{0, \frac{1}{2}\right\}$-Chvátal-Gomory cuts of Caprara \& Fischetti [1996].

We recall these concepts for later comparisons with our approach. We do neither discuss the relation to projection techniques nor to Padberg \& Sung [1991] here; this would blast the scope of this article.

Independence System Approach. An independence system (IS) arises from a set system $\mathfrak{C} \subseteq 2^{V}$ of circuits on a finite ground set $V$ of elements with weights $w_{i}$ for all $i \in V$. A subset $I$ of $V$ is independent if it contains no circuit. The independence system problem (ISP) asks for an independent set of maximum weight. An integer programming formulation of the ISP reads

$$
\begin{array}{ll}
\max & \sum_{i \in V} w_{i} x_{i} \\
\text { (i) } \sum_{i \in C} x_{i} \leq|C|-1 \quad \forall \text { circuits } C \in \mathfrak{C} \\
\text { (ii) } \quad x_{i} \in\{0,1\} \quad \forall i \in V .
\end{array}
$$

The set packing problem, the acyclic subdigraph problem, and the knapsack problem are prominent examples of independence system problems, others, such as the set covering problem, can be transformed into this setting, see, e.g., Laurent [1989] or Nobili \& Sassano [1989]. This means that, in principle, these problems and their associated polytopes can be understood completely in terms of the IS framework.

Facets for the independence system polytope $P_{\mathrm{ISP}}$ include generalized clique, generalized cycle, generalized anticycle, and generalized antiweb inequalities, see, e.g., Nemhauser \& Trotter [1973], Sekiguchi [1983], Euler, Jünger \& Reinelt [1987], Laurent [1989], Nobili \& Sassano [1989]. These results unify and/or extend individual results on special independence system polytopes such as the matroid and the set packing polytope (Nemhauser \& Trotter [1973], Laurent [1989]), the knapsack polytope (Padberg [1975], Laurent [1989]), the acyclic subdigraph polytope (Euler, Jünger \& Reinelt [1987], Nobili \& Sassano [1989]), etc.

On the algorithmic side, virtually no polynomial separation algorithms for general classes of IS inequalities seem to be known. There are, on the contrary, many negative results on the separation of subclasses, e.g., the $\mathcal{N P}$-hardness of the separation of fence inequalities, which happen to be generalized clique inequalities for the acyclic subdigraph polytope, see Müller [1996]. To the best of our knowledge, the only tractable general IS inequalities are those that happen to be polynomial time separable $\left\{0, \frac{1}{2}\right\}$-Chvátal-Gomory cuts, see below.

Transitive Packing Approach. An extended set system $\mathfrak{D} \subseteq 2^{V \times V}$ consists of pairs $(C, \operatorname{tr}(C))$ of (hyper)edges $C \subseteq V$ and associated sets of transitive elements $\operatorname{tr}(C) \subseteq V \backslash C$. $V$ is a finite ground set of elements $v$ with weights $w_{v}$. A subset $I$ of $V$ is a transitive packing $(\mathrm{TP})$ if it contains, for every $(C, \operatorname{tr}(C))$ such that $C \subseteq I$, at least one element from $\operatorname{tr}(C)$. The transitive packing problem (TPP) is to find a transitive packing of maximum weight. An integer programming formulation is

$$
\begin{array}{cc}
\max & \sum_{i \in V} w_{i} x_{i} \\
\text { (i) } \sum_{i \in C} x_{i}-\sum_{i \in \operatorname{tr}(C)} x_{i} \leq|C|-1 \quad \forall(C, \operatorname{tr}(C)) \in \mathfrak{D} \\
x_{i} \in\{0,1\} \quad \forall i \in V .
\end{array}
$$

$(\mathrm{TPP})$

Every ISP with circuit system $\mathfrak{C}$ is a TPP with extended set system $\mathfrak{D}:=\{(C, \emptyset): C \in \mathfrak{C}\}$. This means that transitive packing captures all independence system problems. It subsumes, however, additional combinatorial optimization problems that are not ISPs, among them the clique partitioning problem, the max cut problem, the transitive acyclic subdigraph problem, and the interval order problem, see Müller \& Schulz [1996], Schulz [1996]. 
All types of IS inequalities have been extended to classes of valid inequalities for the transitive packing polytope $P_{\text {TPP }}$. This unifies and/or generalizes results on inequalities for individual transitive packing and related polytopes, among them the clique partitioning polytope and several subpolytopes of the acyclic subdigraph polytope, namely, the transitive acyclic subdigraph polytope, the interval order polytope of a digraph, and the linear ordering polytope, see Müller [1996], Müller \& Schulz [1995, 1996], Schulz [1996].

While the general classes of TP inequalities are as difficult to separate as their IS antecedents, polynomial time separation algorithms are known for interesting subclasses of generalized cycle inequalities for special transitive packing polytopes. Polynomial time separable instances of these weak odd closed walk inequalities (later called weak generalized $(k, 2)$-cycle inequalities) include (and generalize) well studied classes such as the 2-chorded cycle and the odd wheel inequalities for the clique partitioning polytope, see Müller [1996], and most of the known types of inequalities for the linear ordering polytope, see Müller \& Schulz [1995]. Polynomial time separation of weak odd closed walk inequalities carries over to these classes.

$\left\{0, \frac{1}{2}\right\}$-Chvátal-Gomory Cuts. This concept applies to integer linear systems

$$
A x \leq b, \quad x \in \mathbb{Z}^{n},
$$

where $A \in \mathbb{Z}^{m \times n}$ and $b \in \mathbb{Z}^{m}$. A $\left\{0, \frac{1}{2}\right\}$-Chvátal-Gomory cut for IP is an inequality of the form

$$
\lambda^{\mathrm{T}} A x \leq\left\lfloor\lambda^{\mathrm{T}} b\right\rfloor,
$$

where $\lambda^{\mathrm{T}} A \in \mathbb{Z}^{n}$ and $\lambda \in\left\{0, \frac{1}{2}\right\}^{m}$, hence the name. Separation of $\left\{0, \frac{1}{2}\right\}$-Chvátal-Gomory cuts, although $\mathcal{N P}$-hard in general, is polynomial in important cases, e.g., when the system IP has at most two odd coefficients per row, see Caprara \& Fischetti [1996].

$L U$ weakening is a way to proceed when the system IP does not have this property. We may assume that IP contains the bounds $0 \leq x \leq u, u \in(\mathbb{Z} \cup\{\infty\})^{n}$. Denote by $O_{i}:=\left\{1 \leq j \leq n: a_{i j}\right.$ odd $\}$ the index set of the odd coefficients in row $i$ for $i=1, \ldots, m$. To each row $i$ of IP with $\left|O_{i}\right| \geq 3$ one associates $2^{\left|O_{i}\right|\left(\left|O_{i}\right|-1\right) / 2}$ (weaker) inequalities by adding upper and lower bound constraints

$$
\left(A_{i} \cdot x \leq b_{i}\right)+\sum_{j \in L_{i}}\left(-x_{j} \leq 0\right)+\sum_{j \in U_{i}}\left(x_{j} \leq u_{j}\right)
$$

where $\left(L_{i}, U_{i}\right)$ runs through all possible partitions of $O_{i} \backslash\{k, \ell\}$, for all index pairs of odd coefficients $k, \ell \in O_{i}, k \neq \ell$. Note that all of these so-called $L U$ weakenings of row $i$ have exactly 2 odd coefficients. Let $\mathrm{IP}^{\prime}$ be the system that arises from IP by replacing each row with $\left|O_{i}\right| \geq 3$ by all its LU weakenings. IP' has, in general, an exponential number of rows. Caprara \& Fischetti [1996], however, have shown that one can separate in time polynomial in the encoding length of the original system IP over all $\left\{0, \frac{1}{2}\right\}$-ChvátalGomory cuts that can be obtained from its LU weakening $\mathrm{IP}^{\prime}$.

Well known classes of inequalities for combinatorial optimization problems including the clique partitioning problem, the acyclic subdigraph problem, and the asymmetric travelling salesman problem are $\left\{0, \frac{1}{2}\right\}$-Chvátal-Gomory cuts from appropriate IP formulations or their LU weakenings. Whenever IP has polynomial size, these cuts can be separated in polynomial time. This applies, among others, to the 2chorded cycle and the odd wheel inequalities for the clique partitioning polytope, and to a large class of facet defining Möbius ladder inequalities for the linear ordering polytope.

\section{The Acyclic Subdigraph and the Linear Ordering Problem}

Our aim in this section is to construct a set packing relaxation of the acyclic subdigraph and the linear ordering problem in a space of exponential dimension. It will turn out that clique and odd cycle inequalities of this relaxation give rise to (and generalize) several classes of inequalities for the acyclic subdigraph and the linear ordering problem, namely, fence and Möbius ladder inequalities. References are Grötschel, Jünger \& Reinelt [1985b] for the acyclic subdigraph problem and Grötschel, Jünger \& Reinelt [1985a] for the linear ordering problem, see also the monographs Jünger [1985] and Reinelt [1985].

The acyclic subdigraph and the linear ordering problem involve a complete digraph $D_{n}=(V, A)$ on $n$ nodes with integer weights $w_{a}$ on its arcs $a \in A$. An acyclic arc set in $A$ contains no dicycle. The acyclic subdigraph problem (ASP) asks for an acyclic arc set with maximum weight on its arcs. Acyclic arc sets that contain, for any pair of nodes $i$ and $j$, either the $\operatorname{arc} i j$ or the arc $j i$, are called tournaments. 
The linear ordering problem (LOP) is to find a tournament of maximum weight. Integer programming formulations for the ASP and the LOP read as follows:

$$
\begin{aligned}
& \max \sum_{i j \in A} w_{i j} x_{i j} \\
& \sum_{i j \in C} \quad x_{i j} \leq|C|-1 \quad \forall \text { dicycles } C \subseteq A \\
& -x_{i j} \leq 0 \quad \forall i j \in A \\
& x_{i j} \leq 1 \quad \forall i j \in A \\
& x_{i j} \in \mathbb{Z} \quad \forall i j \in A
\end{aligned}
$$

$$
\begin{aligned}
& \max \sum_{i j \in A} w_{i j} x_{i j} \\
& \text { (ii) } \sum_{i j \in C} \quad x_{i j} \leq|C|-1 \forall \text { dicycles } C \subseteq A:|C|=3 \\
& -x_{i j} \leq 0 \quad \forall i j \in A \\
& x_{i j} \leq 1 \quad \forall i j \in A \\
& x_{i j} \in \mathbb{Z} \quad \forall i j \in A .
\end{aligned}
$$

(LOP)

It can be shown that (ASP) is a relaxation of (LOP) and, even more, that the linear ordering polytope $P_{\mathrm{LOP}}$ is a face of the acyclic subdigraph polytope $P_{\mathrm{ASP}}$. In particular, all inequalities that are valid for $P_{\mathrm{ASP}}$ are also valid for $P_{\mathrm{LOP}}$. Two such classes of inequalities for both the ASP and the LOP are the $k$-fence and the Möbius ladder inequalities, see Grötschel, Jünger \& Reinelt [1985b].

A simple $k$-fence involves two disjoint sets of "upper" and "lower" nodes $\left\{u_{1}, \ldots, u_{k}\right\}$ and $\left\{l_{1}, \ldots, l_{k}\right\}$ that are joined by a set of $k$ pales $P_{i}^{\downarrow}:=\left\{u_{i} l_{i}\right\}, i=1, \ldots, k$. The pales are oriented "downward". The $k$-fence is completed by adding all "upward" pickets $P_{i j}^{\uparrow}:=\left\{l_{i} u_{j}\right\}$ with the exception of the antiparallel pales. A (general) $k$-fence is obtained from a simple one by repeated subdivision of arcs, replacing pale and picket arcs by dipaths. Figure 1 shows a simple 4 -fence.

A Möbius ladder consists of an odd number $2 k+1$ of dicycles $C_{0}, \ldots, C_{2 k}$ such that $C_{i}$ and $C_{i+1}$ (indices taken modulo $2 k+1$ ) have a dipath $P_{i}$ in common, see Figure 2 .

Fences and Möbius ladders give rise to valid inequalities for $P_{\mathrm{ASP}}$ : For a $k$-fence $F_{k}$ and a Möbius ladder $M$ of $2 k+1$ dicycles we have

$$
\sum_{i j \in F_{k}} x_{i j} \leq\left|F_{k}\right|-k+1 \quad \text { and } \quad \sum_{i=0}^{2 k} \sum_{i j \in C_{i} \backslash P_{i}} x_{i j} \leq\left(\sum_{i=0}^{2 k}\left|C_{i} \backslash P_{i}\right|\right)-(k+1) .
$$

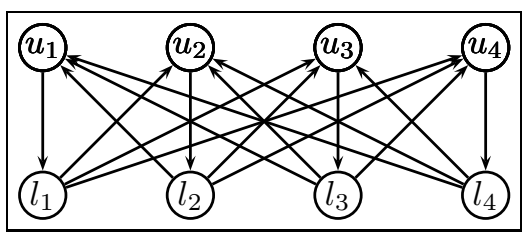

Figure 1: A 4-Fence.

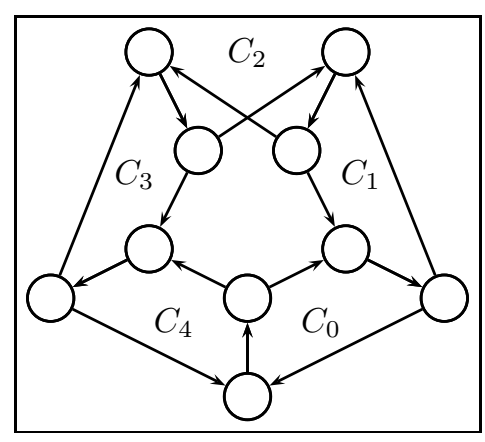

Figure 2: A Möbius Ladder of 5 Dicycles.

A Möbius ladder inequality as above has coefficients larger than one if an arc is contained in more than one of the dipaths $C_{i} \backslash P_{i}$. In this situation of arc repetition, there is a difference to Grötschel, Jünger \& Reinelt [1985b]'s (original) definition, where the coefficients take only values of zero and one, the right hand side is smaller, and the Möbius ladder must meet a number of additional technical requirements to support a valid inequality. The definitions coincide if and only if there is no arc repetition.

We will show now that fences and Möbius ladders are cliques and odd cycles, respectively, in an (exponential) conflict graph $\mathfrak{G}\left(D_{n}\right)=(\mathfrak{V}, \mathfrak{E})$. $\mathfrak{G}$ has the set of all acyclic arc sets of $D_{n}$ as its nodes. We draw an edge $\mathfrak{u} \mathfrak{v}$ between two acyclic arc-set nodes $\mathfrak{u}$ and $\mathfrak{v}$ if their union contains a dicycle. In this case, we say that $\mathfrak{u}$ and $\mathfrak{v}$ are in conflict, because they can not be simultaneously contained in a solution to (ASP).

It is now easy to identify the fences and Möbius ladders with cliques and odd cycles of $\mathfrak{G}$. To obtain a $k$-fence $F_{k}$, we look at the $k$ acyclic arc sets $F_{k}^{i}$ that consist of a pale $P_{i}^{\downarrow}$ and the pickets $P_{i j}^{\uparrow}$ that go up from $l_{i}$, for $i=1, \ldots, k$. We call such a configuration a $k$-fork. Any two forks $F_{k}^{i}$ and $F_{k}^{j}, i \neq j$, are in 

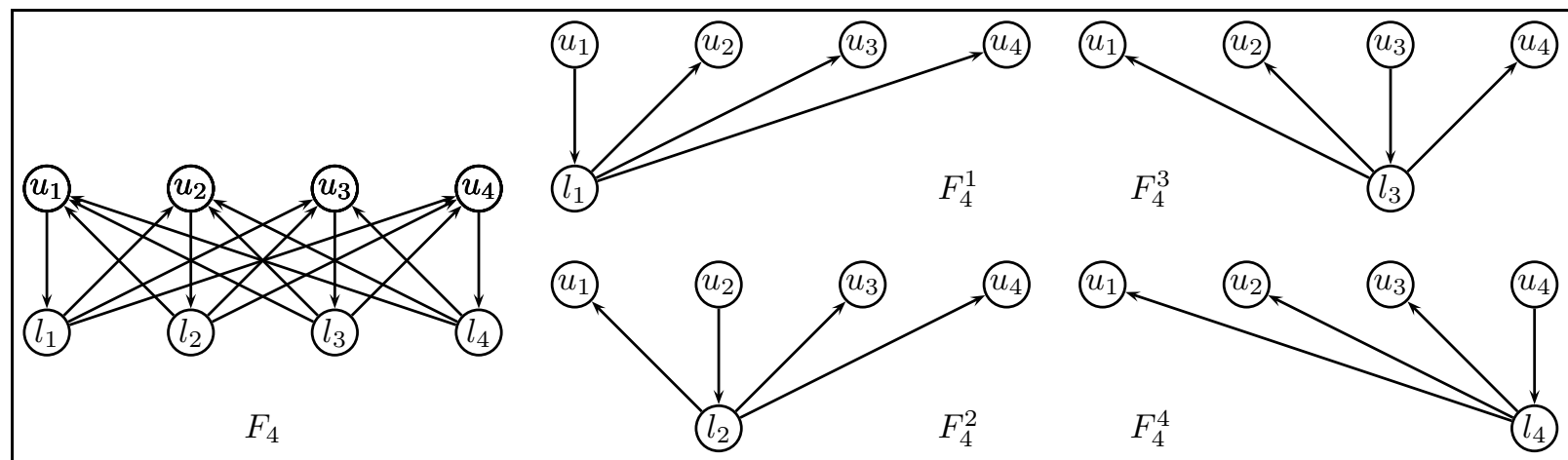

Figure 3: A Fence Clique of Forks.

conflict (they contain a dicycle). Hence, all of them together form a clique in $\mathfrak{G}$. Figure 3 illustrates this construction. Likewise, the Möbius ladders correspond to odd cycles of conflicting dipaths, namely, the dipaths $C_{i} \backslash P_{i}$, see Figure 4.
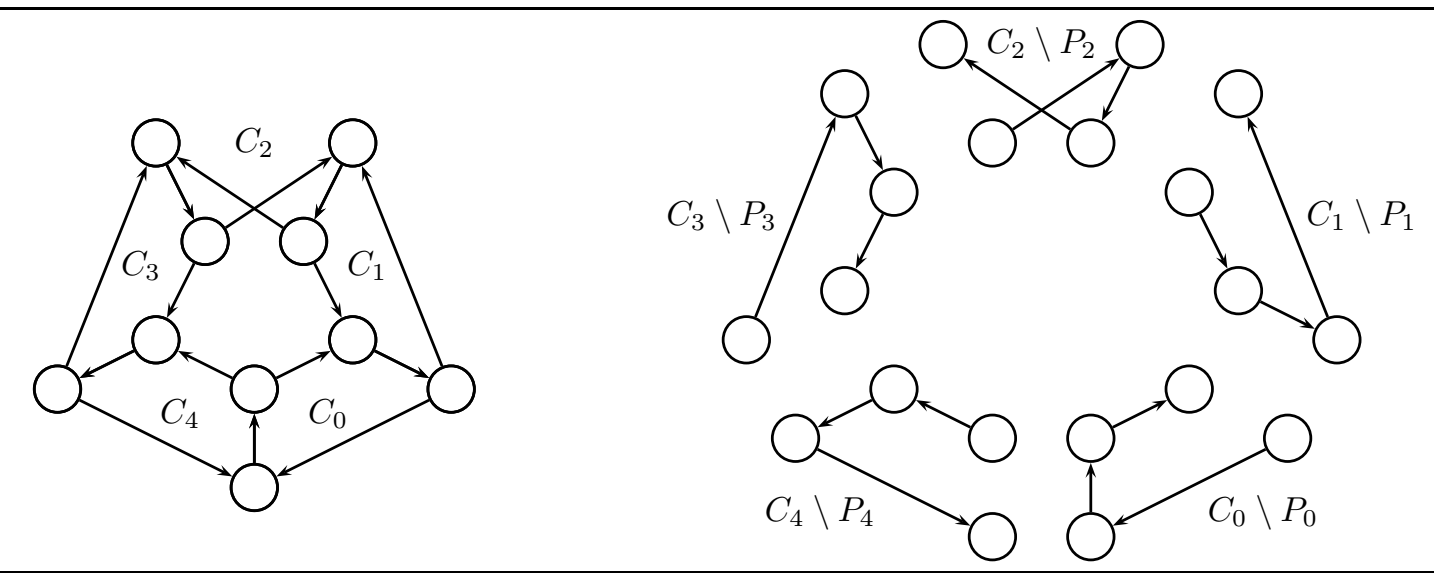

Figure 4: A Möbius Cycle of Dipaths.

The next step to obtain the fence and the Möbius ladder inequalities from the clique and odd cycle inequalities of the (antidominant of the) set packing polytope $\breve{P}_{\mathrm{SSP}}(\mathfrak{G})$ associated with the conflict graph $\mathfrak{G}$, is to construct a set packing relaxation of the ASP. To this purpose, consider the function $\pi: \mathbb{R}^{A} \rightarrow \mathbb{R}^{\mathfrak{V}}$ defined as

$$
\pi_{\mathfrak{v}}(x):=\sum_{i j \in \mathfrak{v}} x_{i j}-(|\mathfrak{v}|-1) \quad \forall \text { acyclic arc sets } \mathfrak{v} \in \mathfrak{V} .
$$

$\pi(x)$ is integral for all integral $x \in \mathbb{R}^{A}$. Moreover, for every incidence vector $x \in P_{\mathrm{ASP}}$ of an acyclic arc set $\operatorname{supp}(x)$ in $D_{n}$, we have that $\pi(x)$ attains its maximum value of one in component $\pi_{\mathfrak{v}}(x)$ if and only if $\mathfrak{v}$ is contained in $\operatorname{supp}(x)$. Since two conflicting acyclic arc sets can not simultaneously be contained in $\operatorname{supp}(x)$, we have that

$$
\mathfrak{u} \mathfrak{v} \in \mathfrak{E} \Longleftrightarrow \pi_{\mathfrak{u}}(x)+\pi_{\mathfrak{v}}(x) \leq 1 \quad \forall x \in P_{\mathrm{ASP}} \cap \mathbb{Z}^{A}
$$

and, by convexity, also for all $x \in P_{\mathrm{ASP}}$. This argument proves that $\breve{P}_{\mathrm{SSP}}(\mathfrak{G})$ is a set packing relaxation of $P_{\text {ASP }}$ in the sense that

3.1 Lemma (Set Packing Relaxation of the ASP) $\pi\left(P_{\mathrm{ASP}}\right) \subseteq \check{P}_{\mathrm{SSP}}\left(\mathfrak{G}\left(D_{n}\right)\right)$.

Note that it is not possible to replace $\breve{P}_{\text {SSP }}$ with $P_{\text {SSP }}$, because the components of $\pi$ can take negative values. More precisely, $\pi(x)$ is in general not the incidence vector of a stable set in $\breve{P}_{\mathrm{SSP}}(\mathfrak{G})$, but $\max \{\mathbf{0}, \pi(x)\}$, with the maximum taken in every component, is. 
Lemma 3.1 allows us to expand an inequality $a^{T} \pi \leq \alpha$ which is valid for $\breve{P}_{\text {SSP }}$ into the inequality $a^{T} \pi(x) \leq \alpha$ which is valid for $P_{\mathrm{ASP}}$. Our next theorem states that, with this terminology, the fence and Möbius ladder inequalities are expansions of clique and odd cycle inequalities, respectively.

\subsection{Theorem (Fence and Möbius Ladder Inequalities)}

Let $D_{n}$ be the complete digraph on $n$ nodes, $P_{\text {ASP }}$ the corresponding acyclic subdigraph polytope, $\mathfrak{G}$ the conflict graph associated with $D_{n}$, and $\breve{P}_{\mathrm{SSP}}(\mathfrak{G})$ the set packing relaxation of $P_{\mathrm{ASP}}$.

(i) Every $k$-fence inequality for $P_{\mathrm{ASP}}$ is the expansion of a clique inequality for $\check{P}_{\mathrm{SSP}}(\mathfrak{G})$.

(ii) Every Möbius ladder inequality for $P_{\mathrm{ASP}}$ is the expansion of an odd cycle inequality for $\breve{P}_{\mathrm{SSP}}(\mathfrak{G})$.

\section{Proof.}

(i) Let $F_{k}$ be a $k$-fence. The forks $F_{k}^{i}, i=1, \ldots, k$, defined on page 5 , are acyclic arc sets and they form a clique in $\mathfrak{G}$, see the discussion on the previous page. An expansion of the corresponding clique inequality yields the desired $k$-fence inequality:

$$
\begin{aligned}
& \sum_{i=1}^{k} \pi_{F_{k}^{i}}(x) \leq 1 \\
\Longleftrightarrow & \sum_{i=1}^{k}\left(\sum_{i j \in F_{k}^{i}} x_{i j}-\left(\left|F_{k}^{i}\right|-1\right)\right)=\sum_{i j \in F_{k}} x_{i j}-\left|F_{k}\right|+k \leq 1 \\
\Longleftrightarrow & \sum_{i j \in F_{k}} x_{i j} \leq\left|F_{k}\right|-k+1 .
\end{aligned}
$$

(ii) Let $M$ be a Möbius ladder consisting of an odd number $2 k+1$ of dicycles $C_{0}, \ldots, C_{2 k}$ such that $C_{i}$ and $C_{i+1}$ have a dipath $P_{i}$ in common. The argument on the previous page showed that the dipaths $C_{i} \backslash P_{i}$ form an odd cycle of $2 k+1$ acyclic arc sets in $\mathfrak{G}$. Expanding the corresponding odd cycle inequality for $\breve{P}_{\mathrm{SSP}}(\mathfrak{G})$, one obtains the Möbius ladder inequality for $M$ :

$$
\begin{aligned}
& \sum_{i=0}^{2 k} \pi_{C_{i} \backslash P_{i}}(x) \leq k \\
\Longleftrightarrow & \sum_{i=0}^{2 k}\left(\sum_{i j \in C_{i} \backslash P_{i}} x_{i j}-\left(\left|C_{i} \backslash P_{i}\right|-1\right)\right) \leq k \\
\Longleftrightarrow & \sum_{i=0}^{2 k} \sum_{i j \in C_{i} \backslash P_{i}} x_{i j} \leq\left(\sum_{i=0}^{2 k}\left|C_{i} \backslash P_{i}\right|\right)-(k+1) .
\end{aligned}
$$

Looking at the separation of Möbius ladder inequalities, we notice that the construction that we just presented to prove Theorem 3.2 (ii) yields a class of odd cycle of dipath inequalities that coincides with the Möbius ladder inequalities as defined in this paper and subsumes Grötschel, Jünger \& Reinelt [1985b]'s Möbius ladder inequalities without arc repetition. Generalizing this class further by allowing the paths $C_{i} \backslash P_{i}$ to intersect themselves on nodes and/or arcs, i.e., by substituting in the definition of a Möbius ladder on page 5 diwalk for dipath and closed diwalk for dicycle, we obtain an even larger class of odd cycle of diwalk inequalities for the acyclic subdigraph polytope. Note that these inequalities do in general not correspond to odd cycles of conflicting acyclic arc sets in the graph $\mathfrak{G}$, because diwalks do not have to be acyclic (they may contain dicycles). This obstacle can be overcome by extending $\mathfrak{G}$ in an appropriate way (including only certain relevant diwalks). At this point, however, we do not want to enter this formalism and defer the details of the extension to the proof of Theorem 3.3.

We can devise a polynomial time separation algorithm for odd cycle of diwalk inequalities, even though the number of diwalks is, in fact, infinite and their length is not even bounded. The idea is to construct a most violated cycle of diwalks out of properly interlinked longest diwalks. Suppose that $M$ is an odd cycle of diwalks (we want to denote these diwalks with a slight extension of our notation by $C_{i} \backslash P_{i}$ ) that induces a violated inequality, and consider the diwalk $P_{i}$ linking the two (successive) closed diwalks $C_{i}$ and $C_{i+1}$. Rearranging, we can isolate the contribution of $P_{i}$ in the constraint as

$$
\left|P_{i}\right|-\sum_{i j \in P_{i}} x_{i j}<\sum_{j \neq i+1}\left(\sum_{i j \in C_{j} \backslash P_{j}} x_{i j}-\left|C_{j} \backslash P_{j}\right|\right)+\sum_{i j \in C_{i+1} \backslash\left(P_{i} \cup P_{i+1}\right)} x_{i j}-\left|C_{i+1} \backslash\left(P_{i+1} \cup P_{i}\right)\right|+(k+1) .
$$


(Here, all sets are supposed to be multisets. We have < because the constraint is, by assumption, violated.) Replacing $P_{i}$ with a diwalk $P$ that has the same endpoints, but is shorter with respect to the length function

$$
|P|-\sum_{i j \in P} x_{i j}=\sum_{i j \in P}\left(1-x_{i j}\right),
$$

we get a more violated cycle of diwalks inequality. If we think of any closed diwalk $C_{i}$ as being composed out of four diwalks, namely, the diwalk $P_{i}^{1}:=P_{i}$ that $C_{i}$ has in common with the succeeding closed diwalk $C_{i+1}$, the diwalk $P_{i}^{2}$ from $P_{i}^{1}$ 's head to the diwalk $P_{i}^{3}:=P_{i-1}$, that $C_{i}$ has in common with the preceding closed diwalk $C_{i-1}$, and the remaining diwalk $P_{i}^{4}$ from $P_{i}^{3}$ 's head to $P_{i}^{1}$ 's tail, the same argument holds for any of these diwalks. This observation allows us to show

\subsection{Theorem (Polynomial Separability of Odd Cycle of Diwalk Inequalities)}

Let $D_{n}$ be the complete digraph on $n$ nodes and $P_{\mathrm{ASP}}$ the associated acyclic subdigraph polytope. Suppose that $x \in \mathbb{Q}^{A}$ satisfies the dicycle and bound constraints (ASP) (ii)-(iv). Then:

Odd cycle of diwalk inequalities violated by $x$ can be separated in polynomial time.

\section{Proof.}

Using Dijkstra's algorithm, we can compute a shortest diwalk $P(u, v)$ with respect to the length (2) from any node $u$ to any node $v$ of $D_{n}$. We can assume these diwalks $P(u, v)$ w.l.o.g. to be of polynomial length (actually we could even assume them to be dipaths). This yields a polynomial number of (2)-shortest diwalks of polynomial length and, moreover, (not every, but) a most violated cycle of diwalks inequality will consist only of these shortest diwalks.

We can find a set of them forming an odd cycle of diwalks as follows. We think of all diwalks $P(u, v)$ as a possible common diwalk $P_{i}$ of two successive closed diwalks $C_{i}$ and $C_{i+1}$ in a cycle of diwalks. To get the diwalks $C_{i} \backslash P_{i}$ as the pieces of the cycle, we compute for any two diwalks $P_{i}$ and $P_{j}$ the (2)-shortest diwalk $P_{i}\left\langle P_{j}\right\rangle$ that starts at $P_{i}$ 's head, contains $P_{j}$, and ends at $P_{i}$ 's tail. Such a diwalk $P_{i}\left\langle P_{j}\right\rangle$ will link (on $P_{j}$ ) properly with another diwalk $P_{j}\left\langle P_{k}\right\rangle$ to form a cycle of diwalks. Computation of the $P_{i}\left\langle P_{j}\right\rangle$ can be performed in polynomial time and yields, in particular, a polynomial number of $n(n-1)(n(n-1)-1)=O\left(n^{4}\right)$ diwalks of polynomial length. Again, (not every, but) a most violated cycle of diwalks inequality will consist only of these diwalks $P_{i}\left\langle P_{j}\right\rangle$.

We can construct a graph that has these diwalks $P_{i}\left\langle P_{j}\right\rangle$ as its nodes with node weights equal to the values $\sum_{i j \in P_{i}\left\langle P_{j}\right\rangle} x_{i j}-\left(\left|P_{i}\left\langle P_{j}\right\rangle\right|-1\right)$ (see (1) on page 6) and that has all edges of the form $\left(P_{i}\left\langle P_{j}\right\rangle, P_{j}\left\langle P_{k}\right\rangle\right)$. A most violated cycle of diwalks inequality corresponds to a most violated odd cycle inequality in the $P_{i}\left\langle P_{j}\right\rangle$-graph. Note that this means, in particular, that there is a most violated cycle of diwalks inequality that consists of a polynomial number of diwalks, even though the total number of diwalks is infinite.

The node weights on an edge in the $P_{i}\left\langle P_{j}\right\rangle$-graph never exceed one because $x$ satisfies the dicycle inequalities (ASP) (ii). Hence, we can find a most violated odd cycle inequality there with the algorithm of Grötschel, Lovász \& Schrijver [1988, Lemma 9.1.11].

\subsection{Corollary (Separation of Möbius Ladder Inequalities)}

A superclass of the Möbius ladder inequalities can be separated in polynomial time.

The same technique can be used for the separation of (general) $k$-fence inequalities for fixed, but arbitrary $k$. Note that one can not enumerate these constraints because general $k$-fences can contain long dipaths. We sketch the construction. Generalizing $k$-forks to $k$-forkings by allowing for arbitrary diwalks as pales and pickets, we can construct classes of clique of $k$-forking inequalities, or even $k$-forking orthonormal representation constraints, that subsume the $k$-fence inequalities. Similar to the proof of Theorem 3.3, one can show that not every, but $a$ most violated clique of $k$-forkings inequality, or $a$ most violated $k$-forking orthonormal representation constraint, will consist of $k$-forkings that are solely composed from (2)-shortest pickets and pales. For fixed $k$, these $k$-forkings can be enumerated in polynomial time and turned into the nodes of a forking conflict graph of polynomial size that has an edge for any two such $k$-forkings $R_{1}$ and $R_{2}$ that contain a closed diwalk of the form "pale $\left(R_{1}\right)$-picket $\left(R_{1}\right)$-pale $\left(R_{2}\right)$-picket $\left(R_{2}\right)$ ". Associating a weight of $x(R)-(|R|-1)$ to each forking-node $R$ (where $R$ is a multiset), we enumerate a most violated $k$-clique or use Grötschel, Lovász \& Schrijver [1988] techniques to separate orthonormal representation constraints. These arguments prove

\subsection{Theorem (Separation of $k$-Fence Inequalities for Fixed $k$ )}

A superclass of the $k$-fence inequalities can be separated in polynomial time for fixed, but arbitrary $k$. 
Fence and Möbius ladder inequalities have been discussed in the contexts of independence systems, transitive packings, and $\left\{0, \frac{1}{2}\right\}$ Chvátal-Gomory cuts in the literature.

Before we start a comparison of results, we want to draw the readers attention to the following subtle difference between the ASP and the LOP. While the length of the dicycles in a facetial Möbius ladder inequality without node and arc repetition for the acyclic subdigraph polytope can be arbitrarily large, the same constraint can only define a facet for the linear ordering polytope if the length of each dicycle is either three or four, see Grötschel, Jünger \& Reinelt [1985b]. This characteristic is responsible for differences in Möbius ladder separation between the LOP and the ASP. No complete facet characterizations for Möbius ladders, neither for $P_{\mathrm{ASP}}$ nor for $P_{\mathrm{LOP}}$, are known in the presence of node and/or arc repetition. Reinelt [1985, Definition 2.4.1] gave the best known sufficient conditions for a Möbius ladder to be a facet of $P_{\mathrm{LOP}}$; the LOP literature focusses on these (Reinelt [1985]'s) Möbius ladders.

Euler, Jünger \& Reinelt [1987] have shown that the (simple) fences are generalized cliques of the independence system of acyclic arc sets of a complete digraph, and that the Möbius ladders without node and arc repetitions are generalized cycles of this IS. In both cases, their arguments prove faceteness of the associated inequalities for $P_{\mathrm{ASP}}$, but they do, per se, not lead to separation algorithms.

Müller \& Schulz [1996] and Schulz [1996] give similar results in the context of transitive packing. They also show that general fences are generalized cliques as well. A slight extension of their cutting plane constructions and inequality classes, with appropriate modifications to allow for arc repetitions, would classify our cliques of forkings as "extended generalized cliques" and our cycles of diwalks as "extended weak generalized $(k, 2)$-cycles".

Müller [1996] has proved that fence separation is $\mathcal{N} \mathcal{P}$-hard. Müller \& Schulz [1995] and Schulz [1996], extending results of Müller [1996], give a polynomial algorithm to separate a superclass (of so-called odd closed walk inequalities) of Reinelt [1985]'s Möbius ladder inequalities for the linear ordering polytope.

Caprara \& Fischetti [1996] exhibit Reinelt [1985]'s Möbius ladder inequalities for the linear ordering polytope as $\left\{0, \frac{1}{2}\right\}$ Chvátal-Gomory cuts from an LU weakening of the system (LOP) (i)-(iv), which is of polynomial size. This already establishes polynomial separability of this class.

To a limited extent, similar results hold for the separation of Möbius ladder inequalities for the acyclic subdigraph polytope. Caprara \& Fischetti [1996] separate a class of inequalities similar to Möbius ladder inequalities (their cut (10)) that consists of $\left\{0, \frac{1}{2}\right\}$ Chvátal-Gomory cuts from an LU weakening of the system (ASP) (ii)-(iv), where (ASP) (ii) is restricted to a polynomial number of dicycle inequalities, e.g., those from dicycles of some arbitrary, but bounded length. In case of arc repetition, Caprara \& Fischetti [1996]'s cut (10) is stronger than a corresponding cycle of diwalks inequality whenever the structure of the latter gives also rise to the former. Möbius ladders with dicycles of arbitrary length, however, can not be separated in this way.

\section{The Clique Partitioning Problem}

In this section, we investigate set packing relaxations of combinatorial optimization problems in connection with cuts: The clique partitioning, the $k$-multicut, and the max cut problem. We will see that the 2 -chorded cycle inequalities for the clique partitioning polytope can be seen as cycles of "lower" triangle inequalities. As a reference to the clique partitioning problem, we suggest Grötschel \& Wakabayashi [1990], see also Wakabayashi [1986], for the multicut and the max cut problem Deza \& Laurent [1997].

The three cut problems of this section come up on a complete graph $K_{n}=(V, E)$ on $n$ nodes with integer weights $w: E \rightarrow \mathbb{Z}$ on its edges. The clique partitioning problem (CPP) is to find a partition of $V$ into an arbitrary number $k$ of cliques $V=C_{1} \cup \ldots \cup C_{k}$ (where $\bullet$ denotes a union of disjoint sets), such that the sum of the weights of the edges that run between different cliques is maximal. In other words, we are trying to find a multicut $\delta\left(C_{1}: \cdots: C_{k}\right)$ of maximum weight, where the number $k$ of (non-empty) members $C_{i}$ of the clique partition $C_{1} \cup \ldots \cup C_{k}$ is arbitrary. One obtains the $k$-multicut problem ( $k$-MCP) from this formulation by restricting the number of cliques to be less than or equal to some given number $k$, and the max cut problem $(\mathrm{MCP})$ by prescribing $k=2$. Thus, any $(\max )$ cut is a $k$-multicut $(k \geq 2)$, and any $k$-multicut comes from a clique partition. We remark that the CPP is commonly stated in an equivalent version to find a clique partition that minimizes the sum of the edge weights inside the cliques.

Denote by $\mathfrak{V}_{\Delta}$ the set of all ordered triples $(i, j, k) \in V^{3}$ of distinct nodes of $K_{n}$, such that, in particular, $(i, j, k)$ forms a triangle. With this notation, integer programs for the CPP and the $k$-MCP read 


$$
\begin{aligned}
x_{i j}-x_{j k}-x_{i k} \leq 0 & \forall(i, j, k) \in \mathfrak{V}_{\Delta} \\
-x_{i j} \leq 0 & \forall i j \in E \\
x_{i j} \leq 1 & \forall i j \in E \\
x_{i j} \in \mathbb{Z} & \forall i j \in E
\end{aligned}
$$$$
\text { (ii) } x_{i j}-x_{j k}-x_{i k} \leq 0
$$

$\forall(i, j, k) \in \mathfrak{V}_{\Delta}$

$\forall i j \in E$

$\forall i j \in E$

$x_{i j} \leq 1$

$x_{i j} \in \mathbb{Z}$ $\forall i j \in E$.

$$
(k-\mathrm{MCP})
$$

Inequalities (CPP) and ( $k$-MCP) (ii) are called "lower" triangle inequalities (their normal vectors are oriented "downward" such that the induced face is on the "downside" of the polytope). Setting $k$ to 2 , inequalities $(k-\mathrm{MCP})$ (i) turn out to be the "upper" triangle inequalities $x_{i j}+x_{j k}+x_{i k} \leq 2$ for all $(i, j, k) \in \mathfrak{V}_{\Delta}$, and (2-MCP) is an integer programming formulation for the max cut problem. For $k=n$, on the other hand, $(k$-MCP) (i) becomes void and $(n-\mathrm{MCP})$ coincides with $(\mathrm{CPP})$. Hence, $(\mathrm{CPP})$ is a relaxation of $(k-\mathrm{MCP})$ which in turn is a relaxation of $(\mathrm{MCP})$ and the associated polytopes $P_{\mathrm{CPP}}, P_{k-\mathrm{MCP}}$, and $P_{\mathrm{MCP}}$ satisfy

$$
P_{\mathrm{CPP}} \supseteq P_{k-\mathrm{MCP}} \supseteq P_{\mathrm{MCP}}
$$

In particular, any valid inequality for the clique partitioning polytope is also valid for the $k$-multicut and the max cut polytope. One such family are the 2-chorded cycle inequalities of Grötschel \& Wakabayashi [1990].

A 2-chorded cycle is an odd cycle $C$ of $K_{n}$ together with its set of 2-chords $\bar{C}$, see Figure 5 . The associated inequality states that

$$
\sum_{i j \in \bar{C}} x_{i j}-\sum_{i j \in C} x_{i j} \leq(|C|-1) / 2
$$

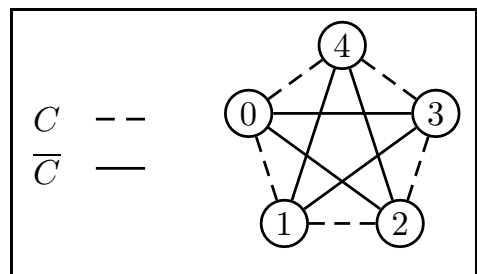

Figure 5: A 2-Chorded Cycle.

We show next that the 2-chorded cycle inequalities arise from odd cycle inequalities of a set packing relaxation of the clique partitioning (or $k$-multicut or max cut) problem. Our arguments establish the polynomial time separability of this class in an alternative way to earlier proofs of Müller [1996] and Caprara \& Fischetti [1996].

The relaxation involves a "lower triangle" conflict graph $\mathfrak{G}_{\Delta}\left(K_{n}\right)=\left(\mathfrak{V}_{\Delta}, \mathfrak{E}_{\Delta}\right) . \mathfrak{V}_{\Delta}$ consists of all ordered triples $(i, j, k) \in V^{3}$ of distinct nodes of $K_{n}$, the edges $\mathfrak{E}$ of $\mathfrak{G}$ are of the form $(i, j, k)(l, i, j),(i, j, k)(l, j, i)$, $(i, j, k)(l, i, k)$, and $(i, j, k)(l, k, i)$ (the meaning of this definition will become clear in a second).

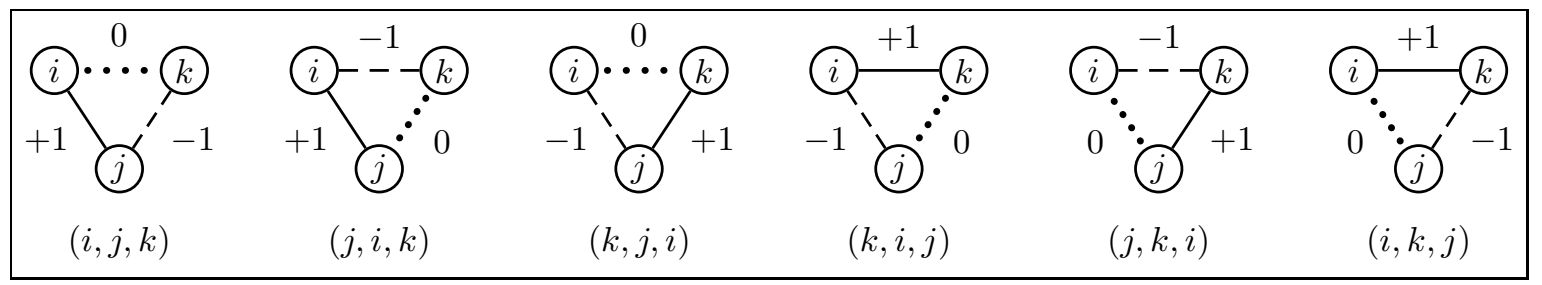

Figure 6: Labeling Lower Triangles.

To construct a set packing relaxation of the clique partitioning problem with this graph, we define a mapping $\pi: \mathbb{R}^{E} \rightarrow \mathbb{R}^{\mathfrak{V}_{\Delta}}$ as

$$
\pi_{(i, j, k)}(x):=x_{i j}-x_{j k} \quad \forall \text { ordered triples }(i, j, k) \in \mathfrak{V}_{\Delta} .
$$


$\pi_{(i, j, k)}(x)$ is integral if $x \in \mathbb{R}^{E}$ is integral. Moreover, for every multicut $x \in P_{\mathrm{CPP}}$, the component $\pi_{(i, j, k)}(x)$ attains its maximum value of one if and only if the nodes $j$ and $k$ belong to the same clique $\left(x_{j k}=0\right)$, but node $i$ does not $\left(x_{i j}=x_{i k}=1\right)$. The reader may think of the triples $(i, j, k)$ as "edge-labelled triangles" as shown in Figure 6; then, it is easy to see that

$$
\mathfrak{u} \mathfrak{v} \in \mathfrak{E}_{\Delta} \Longleftrightarrow \pi_{\mathfrak{u}}(x)+\pi_{\mathfrak{v}}(x) \leq 1 \quad \forall x \in P_{\mathrm{CPP}} \cap \mathbb{Z}^{E}
$$

and thus for all $x \in P_{\mathrm{CPP}}$. In other words, $\mathfrak{E}_{\Delta}$ was defined in such a way that two triples are joined by an edge if and only if it is impossible that both attain their maximum value of one under $\pi$ simultaneously. This argument shows that $P_{\mathrm{SSP}}\left(\mathfrak{G}_{\Delta}\right)$ is a "lower triangle" set packing relaxation of $P_{\mathrm{CPP}}$ :

\subsection{Lemma (Set Packing Relaxation of the CPP) $\pi\left(P_{\mathrm{CPP}}\right) \subseteq P_{\mathrm{SSP}}\left(\mathfrak{G}_{\Delta}\left(K_{n}\right)\right)$.}

The construction is called a "lower triangle set packing relaxation", because one obtains the components $\pi_{(i, j, k)}(x)=x_{i j}-x_{j k} \leq 1$ of $\pi$ from the lower triangle inequalities (CPP) (ii) by setting $x_{i k}=1$ :

$$
x_{i j}-x_{j k}-x_{i k} \leq 0 \Longleftrightarrow x_{i j}-x_{j k} \leq x_{i k} .
$$

We are now ready to state our result that the 2-chorded cycle inequalities are expansions (see the definition on page 7 ) of odd cycle inequalities of $\check{P}_{\mathrm{SSP}}\left(\mathfrak{G}_{\Delta}\right)$.

\subsection{Theorem (2-Chorded Cycle Inequalities)}

Let $K_{n}$ be the complete graph on $n$ nodes, $P_{\mathrm{CPP}}$ the corresponding clique partitioning polytope, $\mathfrak{G}_{\Delta}$ the lower triangle conflict graph, and $\check{P}_{\mathrm{SSP}}\left(\mathfrak{G}_{\Delta}\right)$ the lower triangle set packing relaxation of $P_{\mathrm{CPP}}$.

Every 2-chorded cycle inequality for $P_{\mathrm{CPP}}$ is the expansion of an odd cycle inequality for $\check{P}_{\mathrm{SSP}}\left(\mathfrak{G}_{\Delta}\right)$.

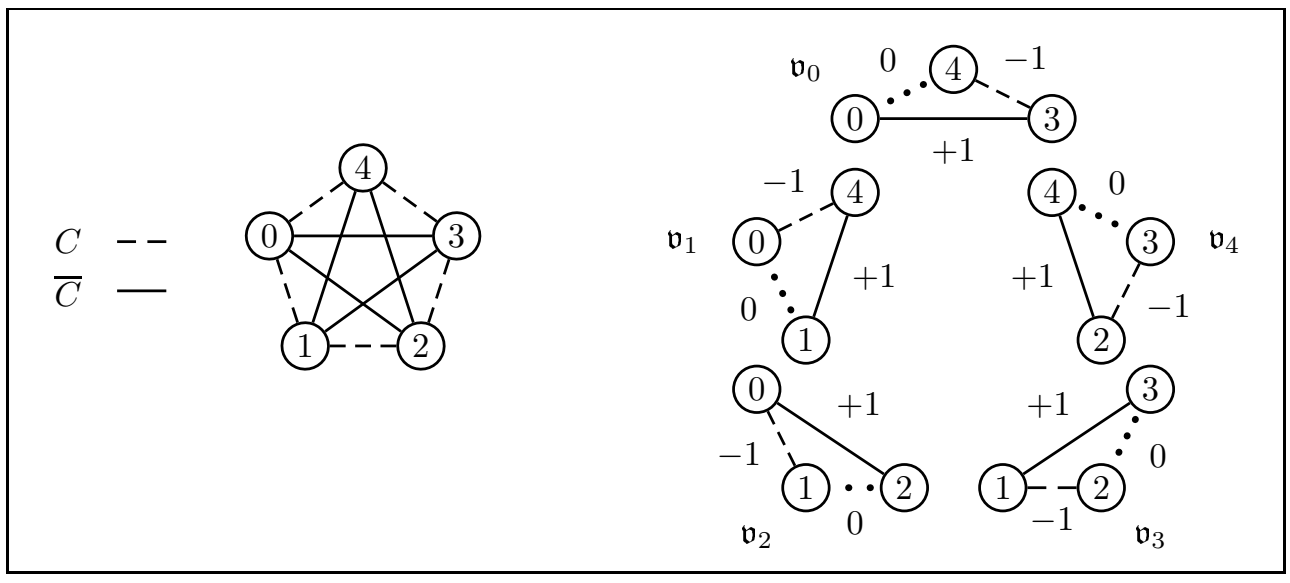

Figure 7: An Odd Cycle of Lower Triangle Inequalities.

\section{Proof.}

Let $C \cup \bar{C}$ be a 2 -chorded cycle in $K_{n}$ with node set $\{0, \ldots, 2 k\}$. By definition, $C=\{i j: i=0, \ldots, 2 k, j=$ $i+1\}$ and $\bar{C}=\{i j: i=0, \ldots, 2 k, j=i+2\}$ (where indices are taken modulo $2 k+1$ ).

Consider the $2 k+1$ triples $\mathfrak{v}_{i}:=(i, i-2, i-1), i=0, \ldots, 2 k$ (indices modulo $2 k+1$ ). One verifies that $\mathfrak{v}_{i} \mathfrak{v}_{i+1} \in \mathfrak{E}$ are in conflict and form the edge set of an odd cycle in $\mathfrak{G}_{\Delta}$, see Figure 7 for an example. The associated odd cycle inequality expands to the 2-chorded cycle inequality in question:

$$
\sum_{i=0}^{2 k} \pi_{(i, i-2, i-1)}(x)=\sum_{i=0}^{2 k} x_{(i, i-2)}-x_{(i-2, i-1)}=\sum_{i j \in \bar{C}} x_{i j}-\sum_{i j \in C} x_{i j} \leq(|C|-1) / 2 .
$$

Calling the expansions of odd cycle inequalities for $\check{P}_{\mathrm{SSP}}\left(\mathfrak{G}_{\Delta}\right)$ inequalities from odd cycles of lower triangle inequalities, we obtain

\subsection{Corollary (Separation of Inequalities from Odd Cycles of Lower Triangle Inequalities)}

Let $K_{n}$ be the complete graph on $n$ nodes and $P_{\mathrm{CPP}}$ the associated clique partitioning polytope. Suppose $x \in \mathbb{Q}^{E}$ satisfies the constraints (CPP) (ii)-(iv). Then:

Inequalities from odd cycles of lower triangle inequalities violated by $x$ can be separated in polynomial time. 
Proof.

The conflict graph $\mathfrak{G}_{\Delta}$ has $6 \times\left(\begin{array}{l}n \\ 3\end{array}\right)=O\left(n^{3}\right)$ triple-nodes. Its size is polynomial. The sum of the node weights on an edge never exceeds one. Applying the algorithm of Grötschel, Lovász \& Schrijver [1988, Lemma 9.1.11], we can find a most violated odd cycle inequality in $\mathfrak{G}_{\Delta}$ in polynomial time.

\subsection{Corollary (Separation of 2-Chorded Cycle Inequalities)}

A superclass of the 2-chorded cycle inequalities can be separated in polynomial time.

Note that the conflicts between two successive triples $\mathfrak{v}_{i}=(i, i-2, i-1)$ and $\mathfrak{v}_{i+1}=(i+1, i-1, i)$ in a 2 -chorded cycle stem from the common edge connecting nodes $i$ and $i-1$, that has a coefficient of -1 in $\pi_{\mathfrak{v}_{i+1}}$ and 0 in $\pi_{\mathfrak{v}_{i}}$. But conflicts arise also from common edges with +1 and -1 coefficients. Thus, besides possible node/edge repetitions and the like, odd cycle of lower triangle inequalities give rise to inequalities that do not correspond to 2-chorded cycle inequalities.

Müller [1996] obtained similar results in a transitive packing context. He showed that the 2-chorded cycles belong to a larger class of odd closed walk inequalities and gave a polynomial time separation algorithm.

Caprara \& Fischetti [1996] derived the 2-chorded cycle inequalities as $\left\{0, \frac{1}{2}\right\}$-Chvátal-Gomory cuts from an LU weakening of the polynomial sized system (CPP) (ii)-(iv), thereby proving their polynomial separability.

\section{The Set Packing Problem}

We have demonstrated in the examples of the preceding sections that certain combinatorial optimization problems have interesting set packing relaxations. Perhaps a bit surprising, we show now that the set packing problem itself also has interesting set packing relaxations! These considerations yield alternative derivation and separation techniques for several classes of wheel inequalities, including two classes introduced by Barahona \& Mahjoub [1994] and Cheng \& Cunningham [1997], as well as a new class of cycle of cycles inequalities. A survey on results for the set packing problem can be found in Grötschel, Lovász \& Schrijver [1988].

The examples of this sections are based on a "rank" set packing relaxation that we introduce now. Given a set packing problem $(\mathrm{SPP})$ on a graph $G=(V, E)$, the associated conflict graph $\mathfrak{G}=(\mathfrak{V}, \mathfrak{E})$ of the relaxation has the set $\mathfrak{V}:=\{G[H]: H \subseteq V\}$ of all node induced subgraphs of $G$ as its nodes. In order to define the set of edges, we consider the mapping $\pi: \mathbb{R}^{V} \rightarrow \mathbb{R}^{\mathfrak{V}}$ defined as

$$
\pi_{G[H]}(x)=\sum_{i \in G[H]} x_{i}-(\alpha(G[H])-1) \quad \forall \text { node induced subgraphs } G[H] \in \mathfrak{V},
$$

where $\alpha(G[H])$ denotes the rank, i.e., the maximum cardinality of a stable set, of $G[H]$. We draw an edge between two subgraphs $G[H]$ and $G[W]$ if there is no stable set in $G$ such that its restrictions to $G[H]$ and $G[W]$ are simultaneously stable sets of maximum cardinality in $G[H]$ and $G[W]$, i.e.,

$$
G[H] G[W] \in \mathfrak{E} \Longleftrightarrow \pi_{G[H]}(x)+\pi_{G[W]}(x) \leq 1 \quad \forall x \in P_{\mathrm{SSP}}(G) \cap \mathbb{Z}^{V}
$$

Well known arguments show that $\check{P}_{\mathrm{SSP}}(\mathfrak{G})$ is a set packing relaxation of $P_{\mathrm{SSP}}$ in the exponential space $\mathbb{R}^{\mathfrak{V}}$ :

5.1 Lemma (Rank Set Packing Relaxation of the SSP) $\pi\left(P_{\mathrm{SSP}}\right) \subseteq \breve{P}_{\mathrm{SSP}}(\mathfrak{G})$.

\subsection{Wheel Inequalities}

One method to derive polynomial time separable expansions of inequalities from the rank relaxation is to consider subgraphs of $\mathfrak{G}$ of polynomial size. A natural idea is to restrict the set of nodes of $\mathfrak{G}$ to

$$
\mathfrak{V}_{k}:=\{G[H]: H \subseteq V:|H| \leq k\},
$$

the node induced subgraphs $G[H]$ of $G$ with bounded numbers of nodes $|H| \leq k$ for some arbitrary, but fixed bound $k$. The smallest interesting case is $k=2$, where $G[H](|H| \leq 2)$ is either empty, a singleton, an edge, or a coedge (complement of an edge). The odd cycle inequalities that one obtains from this restricted relaxation $\breve{P}_{\mathrm{SSP}}\left(\mathfrak{G}\left[\mathfrak{V}_{2}\right]\right)$ contain, among other classes, the odd wheel inequalities of the set packing polytope. A $2 k+1$-wheel is an odd cycle $C$ of $2 k+1$ nodes $\{0 \ldots, 2 k\}$, say, plus an additional node $2 k+1$ that is connected to all nodes of the cycle $C$. $C$ is the rim of the wheel, node $2 k+1$ is the $h u b$, and the edges 
connecting the node $2 k+1$ and $i, i=0, \ldots, 2 k$, are called spokes. For such a configuration, the following inequality holds:

$$
k x_{2 k+1}+\sum_{i=0}^{2 k} x_{i} \leq k
$$

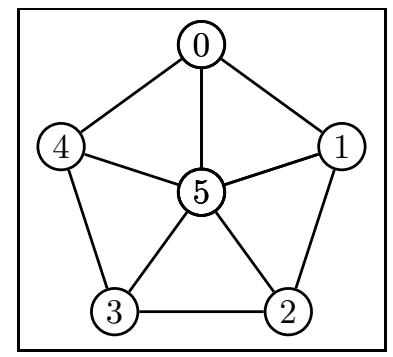

Figure 8: A 5-Wheel.

An odd wheel inequality can be obtained by a sequential lifting of the hub into the odd cycle inequality that corresponds to the rim. This can be used to construct a polynomial time separation algorithm for wheel inequalities which tries all possible hubs. An alternative derivation is

\subsection{Theorem (Odd Wheel Inequalities)}

Let $G=(V, E)$ be a graph, $P_{\mathrm{SSP}}$ the associated set packing polytope, $\mathfrak{G}$ the rank conflict graph, and $\breve{P}_{\mathrm{SSP}}(\mathfrak{G})$ the rank set packing relaxation of $P_{\mathrm{SSP}}$.

Every odd wheel inequality for $P_{\mathrm{SSP}}$ is the expansion of an odd cycle inequality for $\breve{P}_{\mathrm{SSP}}\left(\mathfrak{G}\left[\mathfrak{V}_{2}\right]\right)$.

\subsection{Observation (Separation of Inequalities from Odd Cycles of Nodes, Edges \& Coedges)}

Let $G=(V, E)$ be a graph and $P_{\mathrm{SSP}}$ the associated set packing polytope. Suppose $x \in \mathbb{Q}^{V}$ satisfies all edge constraints $x_{i}+x_{j} \leq 1, i j \in E$, and the bounds $0 \leq x_{i} \leq 1, i \in V$. Then:

Inequalities from odd cycles of nodes, edges, and coedges violated by $x$ can be separated in polynomial time.

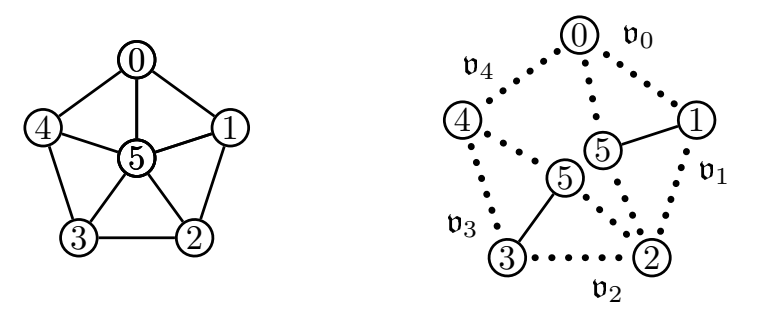

Figure 9: A Cycle of Nodes and Edges.

\section{Proof.}

Consider a $2 k+1$ wheel with $\operatorname{rim} C=\{0, \ldots, 2 k\}$ and hub node $2 k+1$. The subgraphs $\mathfrak{v}_{i}:=G[\{i, 2 k+1\}]$, $i=1,3, \ldots, 2 k-1$, induced by the spokes with odd rim nodes, and the subgraphs $\mathfrak{v}_{i}=G[\{i\}], i=$ $0,2, \ldots, 2 k$, induced by the even rim nodes, form an odd cycle in $\mathfrak{G}$, see Figure 9 (the original wheel is on the left, the nodes of the conflict graph are right, the dotted edges indicate conflicts). Expanding the associated odd cycle inequality yields the wheel inequality:

$$
\sum_{i=0}^{2 k} \pi_{\mathfrak{v}_{i}(x)}=\sum_{i=1,3, \ldots, 2 k-1}\left(x_{i}+x_{2 k+1}\right)+\sum_{i=0,2, \ldots, 2 k} x_{i}=k x_{2 k+1}+\sum_{i=0}^{2 k} x_{i} \leq k .
$$

We show now two examples of cycles of nodes, edges, and coedges that give rise to facetial inequalities that do not correspond to odd wheels. The cycle on the left side of Figure 10 consists of the nodes 0, 2, and 3 and the edges $(1,5)$ and $(4,6)$, the one on the right of the edges $(1,6),(2,7),(3,8)$, and $(4,9)$ and the coedge $(0,5)$. The associated inequalities are

$$
\begin{aligned}
x_{0}+\left(x_{5}+x_{1}\right)+x_{2}+x_{3}+\left(x_{6}+x_{4}\right) & \leq 2 \\
\left(x_{5}+x_{0}-1\right)+\left(x_{6}+x_{1}\right)+\left(x_{7}+x_{2}\right)+\left(x_{8}+x_{3}\right)+\left(x_{9}+x_{4}\right) & \leq 2 \Longleftrightarrow \sum_{i=0}^{9} x_{i} \leq 2
\end{aligned}
$$




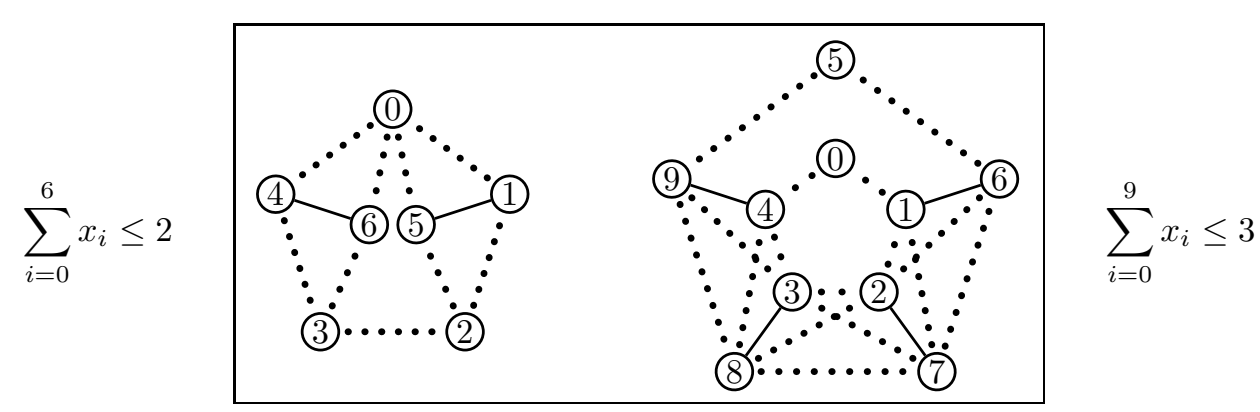

Figure 10: Two Generalizations of Odd Wheel Inequalities.

Another generalization of odd wheel inequalities was given by Barahona \& Mahjoub [1994] and Cheng \& Cunningham [1997]. They introduce two classes of inequalities that have subdivisions of odd wheels as support graphs, where each face cycle must be odd, see Figure 11. Formally, a generalized $2 k+1$-wheel consists of an odd number $2 k+1$ of spoke ( path) $s S_{i}, i=0, \ldots, 2 k$, that all have one common endnode, the hub $h$. The opposite endnodes of any two successive spokes $S_{i}$ and $S_{i+1}$ (indices taken modulo $2 k+1$ ) are joined by a rim path $R_{i}, i=0, \ldots, 2 k$, such that the face cycle formed by $S_{i}, R_{i}$ and $S_{i+1}$ is odd. Following for the remainder of this subsection Cheng \& Cunningham [1997]'s terminology, a spoke is called even and odd if it has an even and odd number of edges (not of nodes!), respectively. (We temporarily override here the node oriented parity definition of the introduction for notational consistency with the literature.) Let $\mathcal{E}$ and $\mathcal{O}$ be the endnodes of the even and odd spokes of an odd wheel $W$ of this kind with some number $2 k+1$ of faces, and let $h$ be the hub. A wheel inequality of type I states that

$$
k x_{h}+\sum_{i \in W \backslash\{h\}} x_{i}+\sum_{i \in \mathcal{E}} x_{i} \leq \frac{|W|+|\mathcal{E}|}{2}-1
$$

A second variant of wheel inequalities (of type II, associated with the same wheel) states that

$$
(k+1) x_{h}+\sum_{i \in W \backslash\{h\}} x_{i}+\sum_{i \in \mathcal{O}} x_{i} \leq \frac{|W|+|\mathcal{O}|-1}{2} .
$$

We remark that these wheels do in general not arise from cycles of subgraphs of bounded size because they contain potentially very long paths.

\subsection{Theorem (Odd Wheel Inequalities)}

Let $G=(V, E)$ be a graph, $P_{\mathrm{SSP}}$ the corresponding set packing polytope, $\mathfrak{G}$ the rank conflict graph, and $\check{P}_{\mathrm{SSP}}(\mathfrak{G})$ the rank set packing relaxation of $P_{\mathrm{SSP}}$.

Every odd wheel inequality of type I and II for $P_{\mathrm{SSP}}$ is the expansion of an odd cycle inequality for $\check{P}_{\mathrm{SSP}}(\mathfrak{G})$.

\section{Proof.}

(i) Wheel inequalities of type I.

The idea of the proof is to obtain the wheel inequality (3) of type I as a cycle of paths, namely, the paths

$$
P_{i}:=S_{i} \cup\left\{\begin{array}{ll}
R_{i}, & \text { if } S_{i+1} \text { is even } \\
R_{i} \backslash S_{i+1}, & \text { if } S_{i+1} \text { is odd }
\end{array}\right\} \backslash\left\{\begin{array}{ll}
\emptyset, & \text { if } i \text { is odd } \\
\{h\}, & \text { if } i \text { is even }
\end{array}\right\}, \quad i=0, \ldots, 2 k,
$$

see Figure 11. By definition, a path $P_{i}$ consists of the spoke $S_{i}$ plus minus the hub depending on $i$, and the full rim path $R_{i}$ if the end node of the next spoke (in clockwise order) is even, or the rim path $R_{i}$ without the end of the next spoke in case this spoke is odd. In this way, the even spoke ends, having a coefficient of two in the wheel inequality, appear in two paths, the odd spoke ends in one. (Recall that in this context a spoke was odd/even if it contained an odd/even number of edges.) It is not hard to see that any two successive paths $P_{i}$ and $P_{i+1}$ are in pairwise conflict: The subpaths $P_{i} \backslash\{h\}$ with the hub removed are all odd and in pairwise conflict, and, likewise, the hub is in conflict with any of these subpaths. The odd cycle inequality corresponding to the paths $P_{i}$ expands into the odd wheel inequality (3): 


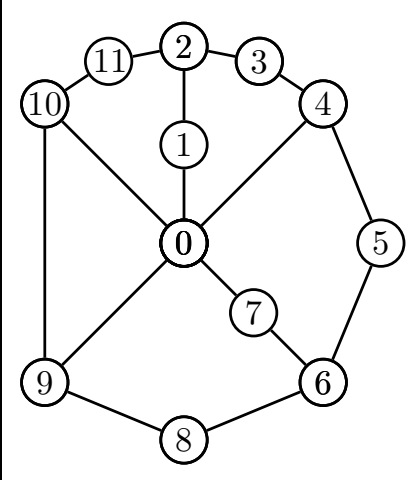

$$
\begin{array}{ll}
\text { hub } & h=0 \\
\text { even spoke ends } & \mathcal{E}=\{2,6\} \\
\text { odd spoke ends } & \mathcal{O}=\{4,9,10\}
\end{array}
$$

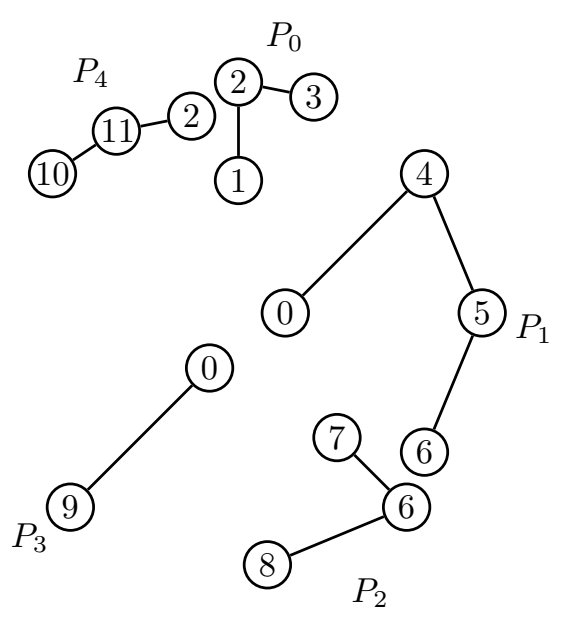

Figure 11: A 5-Wheel and a 5-Cycle of Paths of Type I.

$$
\begin{aligned}
& \sum_{i=0}^{2 k} \pi_{P_{i}}(x) \leq k \\
\Longleftrightarrow & \sum_{i=0}^{k}\left(\sum_{j \in P_{2 i}} x_{j}-\left(\left|P_{2 i}\right|-1\right) / 2\right)+\sum_{i=0}^{k-1}\left(\sum_{j \in P_{2 i+1}} x_{j}-\left(\left|P_{2 i+1}\right|-2\right) / 2\right) \leq k \\
\Longleftrightarrow & k x_{h}+\sum_{j \in W \backslash\{h\}} x_{j}+\sum_{j \in \mathcal{E}} x_{j}-\frac{|W|-1+k+|\mathcal{E}|-(k+1)-2 k}{2} \leq k \\
\Longleftrightarrow & k x_{h}+\sum_{j \in W \backslash\{h\}} x_{j}+\sum_{j \in \mathcal{E}} x_{j} \leq \frac{|W|+|\mathcal{E}|-2 k-2}{2}+k=\frac{|W|+|\mathcal{E}|}{2}-1 .
\end{aligned}
$$

(Here, $\left|P_{i}\right|$ denotes the number of nodes in path $P_{i}$ ).

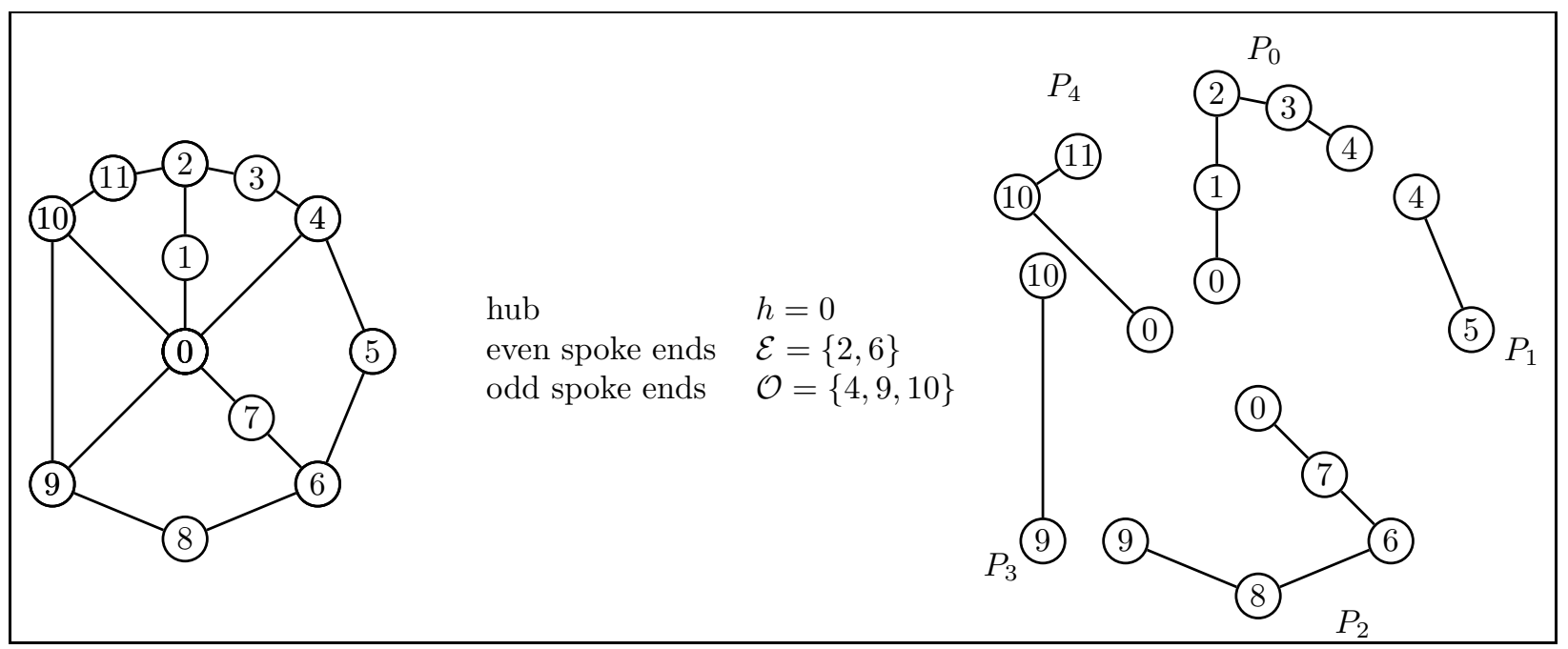

Figure 12: A 5-Wheel and a 5-Cycle of Paths of Type II.

(ii) Wheel inequalities of type II.

The wheel inequalities (4) of type II can be derived in much the same way as their relatives of type I. For 
the sake of completeness, we record the path decomposition

$$
P_{i}:=S_{i} \cup\left\{\begin{array}{ll}
R_{i}, & \text { if } S_{i+1} \text { is odd } \\
R_{i} \backslash S_{i+1}, & \text { if } S_{i+1} \text { is even }
\end{array}\right\} \backslash\left\{\begin{array}{ll}
\emptyset, & \text { if } i \text { is even } \\
\{h\}, & \text { if } i \text { is odd }
\end{array}\right\}, \quad i=0, \ldots, 2 k .
$$

One can verify that, again, any two successive paths are in conflict. A final calculation to expand the resulting odd cycle inequality yields the wheel inequality (4) of type II:

$$
\begin{aligned}
& \sum_{i=0}^{2 k} \pi_{P_{i}}(x) \leq k \\
\Longleftrightarrow & \sum_{i=0}^{k}\left(\sum_{j \in P_{2 i}} x_{j}-\left(\left|P_{2 i}\right|-1\right) / 2\right)+\sum_{i=0}^{k-1}\left(\sum_{j \in P_{2 i+1}} x_{j}-\left(\left|P_{2 i+1}\right|-2\right) / 2\right) \leq k \\
\Longleftrightarrow & (k+1) x_{h}+\sum_{j \in W \backslash\{h\}} x_{j}+\sum_{j \in \mathcal{O}} x_{j}-\frac{|W|-1+(k+1)+|\mathcal{O}|-(k+1)-2 k}{2} \leq k \\
\Longleftrightarrow & (k+1) x_{h}+\sum_{j \in W \backslash\{h\}} x_{j}+\sum_{j \in \mathcal{O}} x_{j} \leq \frac{|W|+|\mathcal{O}|-2 k-1}{2}+k=\frac{|W|+|\mathcal{O}|-1}{2} .
\end{aligned}
$$

One can also derive polynomial time separation algorithms of much the same flavour as for the odd cycle of diwalk inequalities; Cheng \& Cunningham [1997] give such procedures.

\subsection{A New Family of Facets for the Stable Set Polytope}

The rank relaxation of the set packing problem offers ample possibilities to define new classes of polynomially separable inequalities for the set packing problem. We discuss, as one such example, a class of cycle of cycles inequalities.

The way to construct a cycle of cycles inequality is to link an odd number $2 k+1$ of odd cycles $C_{0}, \ldots, C_{2 k}$ to a circular structure, such that any two successive cycles are in pairwise conflict, i.e., $\pi_{C_{i}}(x)+\pi_{C_{i+1}}(x) \leq 1$ (indices taken modulo $2 k+1$ ).

One way to do this is to select from each cycle $C_{i}$ three successive nodes $L_{i} \subseteq C_{i}$ that will serve as a part of the inter-cycle links yet to be formed. The link $L_{i}$ has the property that $\pi_{C_{i}}(x)=1$ implies that at least one of the nodes in $L_{i}$ is contained in the stable set $\operatorname{supp}(x)$, i.e.,

$$
\pi_{C_{i}}(x)=1 \Longrightarrow \sum_{j \in L_{i}} x_{j} \geq 1 \text {. }
$$

If we make sure that any two successive links $L_{i}$ and $L_{i+1}$ are joined by the edge set of the complete bipartite graph $K_{3,3}$, then the inequality

$$
\sum_{j \in L_{i}} x_{j}+\sum_{j \in L_{i+1}} x_{j} \leq 1
$$

holds for all incidence vectors $x$ of stable sets in $G$. But then, the corresponding two successive cycles $C_{i}$ and $C_{i+1}$ are in conflict, i.e., $\pi_{C_{i}}(x)+\pi_{C_{i+1}}(x) \leq 1$, and the cycles $C_{i}$ form an odd cycle in $\mathfrak{G}$, see Figure 13.

\subsection{Theorem (Cycle of Cycles Inequality)}

Let $G=(V, E)$ be a graph and $P_{\mathrm{SSP}}$ be the corresponding set packing polytope. Let $C_{i}, i=0, \ldots, 2 k$, be an odd cycle and $L_{i} \subseteq C_{i}, i=0, \ldots, 2 k$, a set of three successive nodes in $C_{i}$. Assume further that $L_{i}$ and $L_{i+1}, i=0, \ldots, 2 k$, are joined by a complete $K_{3,3}$.

Then the following cycle of cycles inequality is valid for $P_{\mathrm{SSP}}$ :

$$
\sum_{i=0}^{2 k} \sum_{j \in C_{i}} x_{j} \leq \sum_{i=0}^{2 k}\left(\left|C_{i}\right|-1\right) / 2-(k+1) .
$$




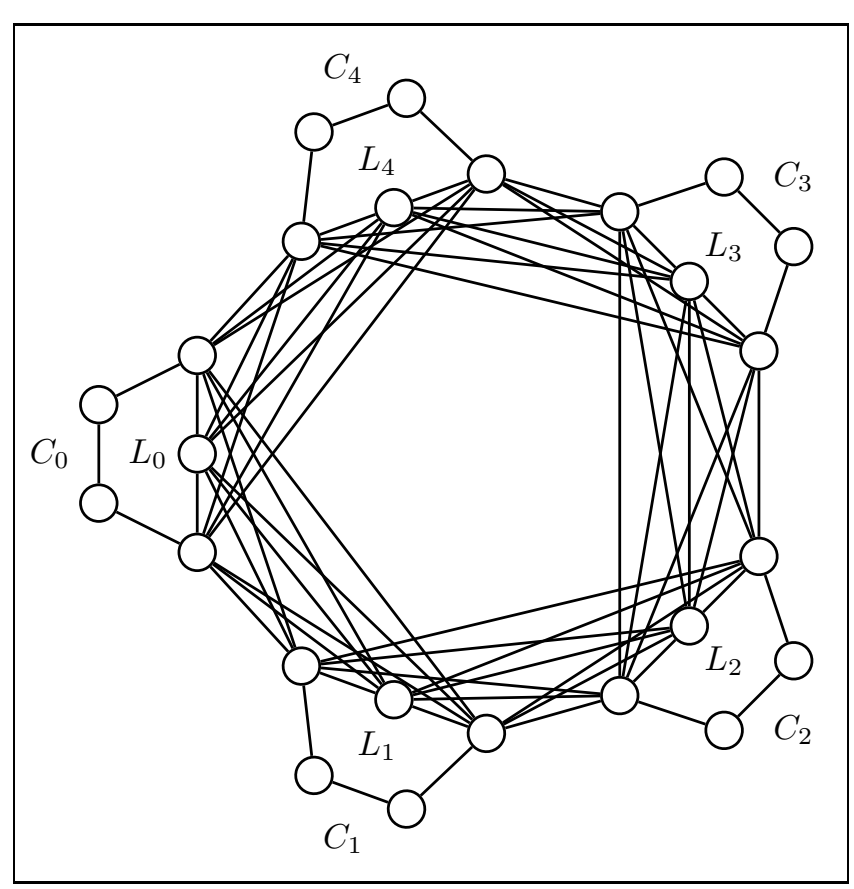

Figure 13: A 5-Cycle of 5-Cycles.

\section{Proof.}

$$
\begin{aligned}
& \sum_{i=0}^{2 k} \pi_{C_{i}}(x) \leq k \\
\Longleftrightarrow & \sum_{i=0}^{2 k}\left(\sum_{j \in C_{i}} x_{j}-\left(\left(\left|C_{i}\right|-1\right) / 2-1\right)\right) \leq k \\
\Longleftrightarrow & \sum_{i=0}^{2 k} \sum_{j \in C_{i}} x_{j} \leq \sum_{i=0}^{2 k}\left(\left(\left|C_{i}\right|-1\right) / 2-1\right)+k=\sum_{i=0}^{2 k}\left(\left|C_{i}\right|-1\right) / 2-(k+1) .
\end{aligned}
$$

A cycle of cycles inequality will in general not be facet inducing, for example, if one of the cycles has a chord that does not join two nodes of its link. But one can come up with conditions that ensure this property. The most simple case is where the cycles $C_{i}$ are holes, all node disjoint, and the only edges that run between different holes belong to the links, i.e., we have a "hole of holes".

\subsection{Theorem (Facet Inducing Cycle of Cyles Inequalities)}

If every cycle in a cycle of cycles inequality is a hole, all node disjoint, and the only edges that run between different holes emerge from the links, then the cycle of cycles inequality is facet inducing.

\section{Proof.}

The proof is based on a sufficiency criterion for the faceteness of rank inequalities by Chvátal [1975]. It is based on the notion of critical edges in a graph $G=(V, E)$ : An edge $i j \in E$ is critical if its removal increases $G$ 's rank, i.e., if $\alpha(G-i j)=\alpha(G)+1$. The criterion states that if the graph $G^{*}:=\left(V, E^{*}\right)$ is connected, where $E^{*}$ is the set of critical edges of $G$, the rank inequality $\sum_{i \in V} x_{i} \leq \alpha(G)$ is facet defining for $P_{\mathrm{SSP}}(G)$. It is easy to see that this condition holds in this case.

\subsection{Theorem (Separation of Cycle of Cycles Inequalities)}

Let $G=(V, E)$ be a graph and $P_{\mathrm{SSP}}$ the associated set packing polytope. Suppose $x \in \mathbb{Q}^{V}$ satisfies all bound, edge, and odd cycle constraints. Then:

Cycle of cycles inequalities violated by $x$ can be separated in polynomial time.

\section{Proof.}

The number of potential links $L_{i}$ is polynomial of order $O\left(|V|^{3}\right)$. We set up a link graph, that has the links 
as its nodes; this device will, in a second, turn out to be a subgraph of $\mathfrak{G}$. Two links are connected by an edge if and only if they are joined by a $K_{3,3}$. To assign weights to the links, we calculate for each link $L_{i}$ the shortest even path $P_{i}$ in $G$ that connects the two endpoints of the link (see, e.g., Barahona \& Mahjoub [1985] how to find even paths); here, shortest means shortest with respect to the length function

$$
\left(1-x_{i}-x_{j}\right) / 2 \quad \forall \text { edges } i j \in E .
$$

$L_{i} \cup P_{i}$ forms an odd cycle $C_{i}$ through $L_{i}$. We set the weight of link $L_{i}$ to the value $\pi_{C_{i}}(x)$, obtain the link graph as a subgraph of $\mathfrak{G}\left[\left\{C_{i}\right\}\right]$ (some edges that correspond to "non-link conflicts" are possibly missing), and detect a violated odd cycle inequality in the link graph if and only if a violated cycle of cycles inequality in $G$ exists.

\section{Acknowledgement}

We thank Adam Letchford, Akiyoshi Shioura, and four anonymous referees for helpful suggestions and comments.

\section{References}

Balas \& Clausen (Eds.) (1995). Integer Programming and Combinatorial Optimiziation, Proc. of the 4th Int. IPCO Conf.

Balas \& Pulleyblank (1989). The Perfectly Matchable Subgraph Polytope of an Arbitrary Graph. Combinatorica 9, 321-32\%.

Barahona \& Mahjoub (1994). Compositions of Graphs and Polyhedra II: Stable Sets. SIAM J. on Disc. Math. 7, 359-371.

Barahona \& Mahjoub (1985). On the Cut Polytope. Math. Prog. 36, 157-173.

Caprara \& Fischetti (1996). \{0, $\left.\frac{1}{2}\right\}$-Chvátal-Gomory Cuts. Math. Prog. 74(3), 221-235.

Cheng \& Cunningham (1997). Wheel Inequalities for Stable Set Polytopes. Math. Prog. 77(3), 389-421.

Chopra \& Rao (1994a). The Steiner Tree Problem I: Formulations, Compositions, and Extensions of Facets. Math. Prog. 64A(2), 209-229.

— (1994b). The Steiner Tree Problem II: Properties and Classes of Facets. Math. Prog. 64A(2), 231-246.

Chvátal (1975). On Certain Polytopes Associated with Graphs. J. Comb. Theory 18, 138-154.

Cunningham, McCormick \& Queyranne (Eds.) (1996). Integer Programming and Combinatorial Optimization, Proc. of the 5th Int. IPCO Conf., Vancouver, British Columbia, Canada.

Deza \& Laurent (1997). Geometry of Cuts and Metrics. Springer Verlag, Berlin.

Euler, Jünger \& Reinelt (1987). Generalizations of Cliques, Odd Cycles and Anticycles and Their Relation to Independence System Polyhedra. Math. of OR 12(3), 451-462.

Grötschel, Jünger \& Reinelt (1985a). Facets of the Linear Ordering Polytope. Math. Prog. 33, 43-60. (1985b). On the Acyclic Subgraph Polytope. Math. Prog. 33, 28-42.

Grötschel, Lovász \& Schrijver (1988). Geometric Algorithms and Combinatorial Optimization. Springer Verlag, Berlin.

Grötschel \& Wakabayashi (1990). Facets of the Clique Partitioning Polytope. Math. Prog. 47(3), 367-387.

Jünger (1985). Polyhedral Combinatorics and the Acyclic Subdigraph Problem, volume 7 of Res. and Exposition in Math. Heldermann Verlag, Berlin.

Laurent (1989). A Generalization of Antiwebs to Independence Systems and Their Canonical Facets. Math. Prog. 45, 97-108.

Lovász \& Schrijver (1991). Cones of Matrices and Set-Functions and 0-1 Optimization. SIAM J. on Opt. 1, $166-190$.

Müller (1996). On the Partial Order Polytope of a Digraph. Math. Prog. 73(1), 31-49.

Müller \& Schulz (1995). The Interval Order Polytope of a Digraph. In Balas \& Clausen [1995], pp. 50-64.

— (1996). Transitive Packing. In Cunningham, McCormick \& Queyranne [1996], pp. 430-444. 
Nemhauser \& Trotter (1973). Properties of Vertex Packing and Independence System Polyhedra. Math. Prog. 6, 48-61.

Nobili \& Sassano (1989). Facets and Lifting Procedures for the Set Covering Polytope. Math. Prog. 45, 111-13\%.

Padberg (1973). On the Facial Structure of Set Packing Polyhedra. Math. Prog. 5, 199-215. (1975). A Note on Zero-One Programming. Op. Res. 23(4), 833-837.

Padberg \& Sung (1991). An Analytical Comparison of Different Formulations of the Travelling Salesman Problem. Math. Prog. 52(2), 315-35\%.

Pulleyblank \& Shepherd (1993). Formulations for the Stable Set Polytope of a Claw-Free Graph. In Rinaldi \& Wolsey [1993], pp. 267-279.

Reinelt (1985). The Linear Ordering Problem: Algorithms and Applications, volume 8 of Res. and Exposition in Math. Heldermann Verlag, Berlin.

Rinaldi \& Wolsey (Eds.) (1993). Integer Programming and Combinatorial Optimiziation, Proc. of the 3rd Int. IPCO Conf.

Schulz (1996). Polytopes and Scheduling. PhD thesis*, Tech. Univ. Berlin.

Sekiguchi (1983). A Note on Node Packing Polytopes on Hypergraphs. OR Letters 2(5), 243-247.

Wakabayashi (1986). Aggregation of Binary Relations: Algorithmic and Polyhedral Investigations. PhD thesis, University of Augsburg.

${ }^{*}$ Avail. at URL ftp://ftp.math.tu-berlin.de/pub/Preprints/combi/ 